\section{Virus infection of baboons}

SIR - Translation of baboon bone marrow is being considered for restoring the immune system of HIV patients ${ }^{1}$. Concern has been expressed that such transplantation could result in the emergence of lethal new viruses in the human population. In this respect, simian haemorrhagic fever virus (SHFV) should be seriously considered as a threat.

SHFV is a member of an as-yet-unnamed new family of positive-stranded RNA viruses $^{2}$. SHFV is endemic in populations of three genera of African monkeys baboons (Papio anuibus and $P$ cyanocephalus), patas monkeys (Erythrocebus patas) and African green monkeys (Ceropithecus aethiops). SHFV seems to establish persistent, perhaps lifelong infections in these monkeys without causing any clinical symptoms. However, artificial transmission of SHFV to Asian monkeys of another genus, namely rhesus monkeys (Macacca mulatta, M. arctoides and $M$. fascicularis), results in haemorrhagic fever that is generally fatal within 2 weeks after infection. Devastating epizootics among rhesus monkeys have occurred in monkey colonies in the former Soviet Union in 1967, the United States in 1967, 1972 and 1989 and in England between 1966 and 1969 with the loss of 1,000-2,000 monkeys. During the 1989 epizootic, which involved three monkey facilities in the United States, Ebola virus was also detected in some monkeys ${ }^{3}$ as discussed in the book The Hot Zone, by Richard Preston (Random House/ Doubleday, 1994) but it has not been resolved to what extent the rhesus monkeys died from infection by SHFV or Ebola virus. This is a definitive example of a transmission of a virus that causes only asymptomatic infections in its natural primate hosts, including baboons, to primates of another genus with devastating consequences.

There is no indication that any humans became infected with SHFV during any of the SHFV epizootics. However, transmission of SHFV from African to Asian monkeys seems to occur only through accidental transfer of blood; initial transmission in epizootics is believed to have occurred by mechanical means through the use of unsterilized syringes. In contrast, transmission between rhesus monkeys is through the respiratory route and is very efficient. Unfortunately, very little information is available on the prevalence of SHFV in African monkey populations and detection of the virus is problematic. SHFV isolates generally grow poorly in cell cultures and infected monkeys often have only low levels of antiviral antibodies. The most sensitive method of detection is inoculation into rhesus monkeys, but this method is very expensive. It is obvious that transplantation of humans with tissues from SHFV-infected baboons would result in transfer of considerable amounts of the virus and could result in selection of a variant that can replicate in humans.

\section{Peter G. W. Plagemann}

Department of Microbiology,

University of Minnesota,

Minneapolis, Minnesota 55455, USA

1. Lehrman, S. Nature 376, 8 \& 204 (1995).

2. Plagemann, P.G.W. Moennig, V. Adv. Virus. Res. 41 99-192 (1992)

3. Jahrling, P.B., Geisberg, T.W., Dalgard, D.W. et al. Lancet 335, 502-505 (1990).

\section{Benefits of placebos}

SIR - Recent correspondence shows that placebo-controlled trials have their drawbacks. Beatrice Golomb ${ }^{1}$ suggests that studies should be carried out to test for possible specific effects of placebo agents so as to eliminate an eventual confounding factor in the interpreting of double-blind study results. Justifiable as it seems from a scientific point of view, this does not appear to me a cost-effective approach. Recent developments show that the costs of new drugs that come to the market will not be automatically reimbursed by health assurance systems. In many countries, the market prices of drugs for one and the same indication are compared and drugs with the lowest daily costs are privileged. The need for extra studies to test for possible specific effects of placebos will increase drug development costs and thus market price. An alternative would be not to use placebos for the control of clinical studies, or to accept small effects of a placebo where its use cannot be circumvented. There may be good reasons to use no placebos for the control of clinical studies ${ }^{2}$. Usually, however, two or more placebo-controlled (pivotal) studies are required to convince the medical profession and health authorities of the therapeutic value of a drug for a readily identifiable category of patients.

As Golomb rightly states, an apparent positive, negative or null effect of a drug may be the consequence of a negative, positive or same-direction effect of a placebo with which it is compared. Because it is not realistic to expect a pharmaceutical company to invest a huge amount of money in the development of a drug that is not viable, false negative effects are unlikely to occur and, if they occur, are taken for granted. Similarly, a lack of significant difference between active and placebo treatment is always a reason to abandon a project and not to invest more money just to confirm whether the placebo is as (in)effective as the (in)active drug. In the worst case, a drug is credited for effects that it does not produce because by chance there had been negative effects of the placebo(s) used in the pivotal studies. Even then, this may not be harmful. It is generally assumed that when there is a moderate difference between two treatments in their effects on some specific disease outcome, this difference might be larger or smaller in other patients (studies), but it is unlikely to be reversed ${ }^{3}$. Besides, a compound with no specific effect (a placebo), but efficacious in the treatment of a disease when adequately administered and free of any toxicity, may be the best 'drug' that one can imagine.

Joep H. Schoemaker

Department of International

Clinical Research,

Roche Nederland BV,

3641 RR Mijdrecht, The Netherlands

1. Golomb, B. Nature 375, 530 (1995).

2. Rothman, K.J. \& Michels, K.B. N. Engl. J. Med 331(6), 394-398 (1994).

3. Peto R. Collins R \& Gray R. J. clin. Epidem. 48(1), 23-40 (1995).

\section{Science in Israel}

SIR-Your supplements on science in different countries are to be commended, and that on science in Israel (Nature 376, $717-732 ; 1995)$ is no exception. Israel harbours a promising potential in science in many various fields, from the computer sciences, through energy sources and water desalination to desertification, which has been augmented by the arrival of immigrant scientists from the former Soviet Union.

But there is a growing concern about the future of science and research and development (R\&D) in Israel especially in basic science. This includes the state of science, fields of activities, centres of excellence and the shaping of science in the future, the cost of research, 'big science' and international cooperation, funding and the need for central (government) support both nationally and internationally; higher education, present and future, particularly with the call for higher education for all. Basic science is a major component of $\mathrm{R} \& \mathrm{D}$ in Israel, chiefly in versatility and output, although applied and industrial R\&D surpass basic science in expenditure and manpower (scientists and engineers). Israel is first in the world in the number of scientific papers published per person.

Your supplement dwelt entirely upon applied and industrial-related R\&D. I hope you will be able to write about basic science in Israel in some future issue.

Yossi Segal

Israel Academy of Sciences

and Humanities,

POB 4040,

Jerusalem 91040, Israel 